\title{
The ASTRI Mini-Array Core Science Program
}

\author{
Stefano Vercellone ${ }^{a, *}$ for the ASTRI Project ${ }^{b}$ \\ ${ }^{a}$ INAF Osservatorio Astronomico di Brera, \\ Via E. Bianchi, 46, I-23807 Merate (LC), Italy \\ ${ }^{b}$ http://www.astri.inaf.it/en/library/ \\ E-mail: stefano.vercellone@inaf.it
}

The portion of the electromagnetic spectrum above a few Teraelectronvolt $(\mathrm{TeV})$ is currently being investigated by means of both ground-based imaging atmospheric Cherenkov telescopes and water Cherenkov detector arrays. In a few years, an array composed of at least nine ASTRI dual-mirror, Schwarzschild-Couder telescopes will be deployed and start scientific observations at the Observatorio del Teide (Tenerife, Spain). The ASTRI Mini-Array will devote the first three to four observing years to specific science topics, with the aim of providing robust answers to a few selected open questions in the very high-energy (VHE, $\mathrm{E}>0.1 \mathrm{TeV}$ ) domain. We identified the following Core Science topics, a.k.a. the "Science Pillars", to be investigated: the origin of cosmic rays, the extra-galactic background light and the study of fundamental physics, the novel field in the VHE domain of gamma-ray bursts and other multi-messenger transients, and finally the usage of the ASTRI Mini-Array to investigate ultra high-energy cosmic rays and to address stellar intensity interferometry studies. We review the results obtained by means of dedicated scientific simulations, proving the potential of the ASTRI Mini-Array in pursuing breakthrough discoveries and discuss the synergies with current and future VHE facilities.

$37^{\text {th }}$ International Cosmic Ray Conference (ICRC 2021)

July 12 th $-23 r d, 2021$

Online - Berlin, Germany

\footnotetext{
${ }^{*}$ Presenter
} 


\section{The ASTRI Mini-Array}

Celestial sources emitting photons above a few tens of $\mathrm{GeV}$ can be detected by means of imaging atmospheric Cherenkov telescopes (IACTs). Currently, H.E.S.S. [1], MAGIC [2], and VERITAS [3] are the major IACTs operating in the Southern (H.E.S.S.) and in the Northern (MAGIC and VERITAS) hemispheres. The energy range that can be explored extends up to a few tens of TeV. An alternative detection tecnique consists of water Cherenkov detectors arrays (WCDAs). HAWC [4], LHAASO [5] and Tibet AS $\gamma$ [6] are the current arrays which allows us to investigate energies up to several hundreds of $\mathrm{TeV}$, reaching the $\mathrm{PeV}$ limit.

The Cherenkov Telescope Array Observatory [CTAO, 7] will be the next large scale Cherenkov array deployed in both hemispheres and covering an energy range from a few tens of $\mathrm{GeV}$ up to a few hundreds of $\mathrm{TeV}$ by means of telescopes of different sizes [see e.g., 8]. A few telescope prototypes were developed in the recent years, among them the ASTRI-Horn dual-mirror, SchwarzschildCouder telescope [9], currently operating on Mount Etna in Sicily (Italy), which obtained the firstlight optical qualification by means of observation of Polaris, using a dedicated optical camera [10], and the first detection of very high-energy $\gamma$-ray emission from the Crab Nebula by a Cherenkov telescope in dual-mirror Schwarzschild-Couder (SC) configuration [11].
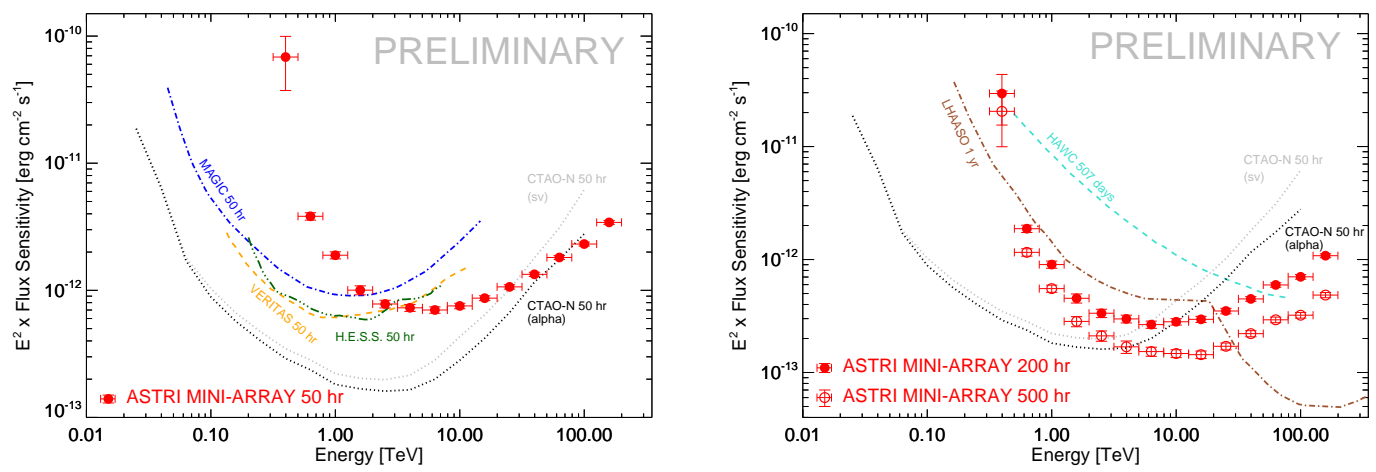

Figure 1: Left Panel: ASTRI Mini-Array on-axis differential sensitivity for $50 \mathrm{hr}$ integration compared with those of current IACTs. Right Panel: ASTRI Mini-Array $200 \mathrm{hr}$ and $500 \mathrm{hr}$ on-axis differential sensitivity compared with those of current WCDAs in the northern hemisphere. Both panels adapted from [12]. CTAO North differential sensitivity curves come from [8, (alpha configuration, 2028)] and [13, (science verification, sv, 2025)], respectively.

The focus of this Work is on the ASTRI Mini-Array [14], which is an array of nine (slightly modified) ASTRI telescopes which will be deployed at the Observatorio del Teide (Canary Island, Spain) at the end of 2021, and will start scientific operation in 2024. We can summarize the main ASTRI Mini-Array performance as follows: field of view of about $10^{\circ}$; energy range from $1 \mathrm{TeV}$ up to $200 \mathrm{TeV}$; angular resolution $\sim 3^{\prime}$ at $10 \mathrm{TeV}$; energy resolution $\sim 10 \%$ at $10 \mathrm{TeV}$. A detailed description of the ASTRI Mini-Array performance is provided in [15].

Figure 1 (left panel) shows the ASTRI Mini-Array on-axis differential sensitivity (50 integration time) compared with those of current and planned IACTs. The differential sensitivity curves 
come from [16, MAGIC], the VERITAS official website ${ }^{1}$, and [17, sensitivity curve for H.E.S.S.-I, stereo reconstruction]. On the right panel, we plot the ASTRI Mini-Array on-axis differential sensitivity compared with those of current WCDAs in the northern hemisphere. The integration times are $200 \mathrm{hr}$ and $500 \mathrm{hr}$ for the ASTRI Mini-Array (typical for a very deep pointing during the core science phase) and about $1 \mathrm{yr}$ for WCDAs, respectively. The differential sensitivity curves come from [18, HAWC], and [19, LHAASO]. We also plotted for comparison the CTAO North differential sensitivity curves for $50 \mathrm{hr}$ integration time both in the "alpha" [(2028, 4 LSTs + 9 MSTs), 8] and in the "science verification" [(2025, 4 LSTs + 5 MSTs), 13] configurations, respectively. The ASTRI Mini-Array differential sensitivity will improve the current IACTs one above a few $\mathrm{TeV}$, and will be competitive with respect to the CTAO North one during the science verification phase. We note that the ASTRI Mini-Array performance remains almost stable across the $10^{\circ}$ field of view, with a degradation at its edge of a factor of about two. On the other side, the WCDAs have $2 \mathrm{sr}$ field of view, but their energy and angular resolution in the same energy range as the ASTRI Mini-Array (about $10 \mathrm{TeV}$ ) are at least a factor of 3-4 worse, making the ASTRI Mini-Array extremely competitive in studying the morphology of extended sources, crowded fields, and accurately monitoring several sources each pointing. In this Work we take into account the ASTRI Prod1-Teide Monte Carlo production described in [12].

\section{Core Science Program}

We plan to devote the first four years of observations to specific science topics with the aim to provide robust answers to a few well-defined open questions, while the second four years of operation will be devoted to observatory science [see 20, 21]. We base our Core Science Program on "Pillars", i.e. science fields in which the ASTRI Mini-Array will contribute breakthrough pieces of evidence to vastly improve our understanding of the a few key science questions. We will investigate the origin of cosmic rays, in particular, we will tackle the long-standing quest of sources which could accelerate hadrons up to petaelectronvolt energies. The ASTRI Mini-Array will be fundamental in cosmology and fundamental physics, by studying the infra-red component of the extra-galactic background light (EBL), as well as by making use of blazars as probes for the axion-like particle and hadron-beam or Lorentz-invariance violation studies. The very recent detections of emission up to a few TeV from GRB 190114C [22] opened a new window in the GRB \& time-domain astrophysics. Finally, The ASTRI Mini-Array will investigate not only $\gamma$-ray sources, but will also explore the direct measurements of cosmic rays and perform stellar intensity interferometry studies.

The origin of cosmic rays. This topic is covered in detail in [23], therefore we will mainly discuss a few recent findings where the ASTRI Mini-Array will provide a significant contribution. Recently, the LHAASO Collaboration [24] reported on the discovery of 12 Galactic sources as possible PeVatron candidates, i.e. sources able to accelerate protons up to $\sim 10^{15} \mathrm{eV}$. Although the highest photons can reach energies of the order of several hundreds of $\mathrm{TeV}$, up to $1.4 \mathrm{PeV}$, the majority of sources are diffuse $\gamma$-ray structures with angular extensions up to $1^{\circ}$. The actual sources responsible for the ultrahigh-energy $\gamma$-ray emission have not yet been univocally identified (except

\footnotetext{
${ }^{1}$ https://veritas.sao.arizona.edu
} 
for the Crab Nebula), leaving open the origin of these extreme accelerators. This discovery is extremely important for the ASTRI Mini-Array science, especially because of its angular resolution which, at energies of about $100 \mathrm{TeV}$, is a factor 3-4 times better in radius than the LHAASO one: $0.08^{\circ}$ vs. $0.24^{\circ}-0.32^{\circ}$. The ASTRI Mini-Array will investigate these and future PeVatron sources providing important information on their morphology in the (10-100) TeV energy range. Figure 2, left panel, shows the ASTRI Mini-Array $200 \mathrm{hr}$ simulation of the region around the the Galactic source 2HWC J1908+063 [VER J1907+062, see also 25, for morphological details]. This is one of the possible PeVatron counterparts of the newly-detected source LHAASO J1908+0621. The light green circle marks the $\sim 0.52^{\circ}$ HAWC error-box (for $E>56 \mathrm{TeV}$ ) [26]. The details of the ASTRI Mini-Array simulations are reported in [12].
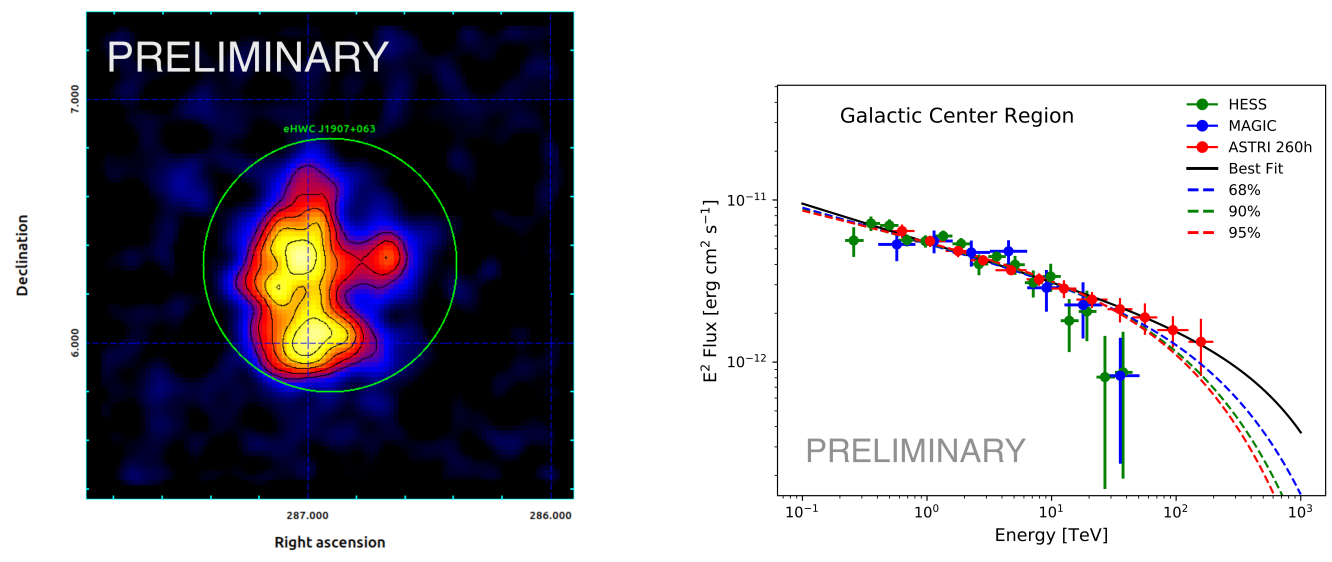

Figure 2: Left Panel: ASTRI Mini-Array $200 \mathrm{hr}$ simulation of the region of the Galactic source 2HWC J1908+063. The light green circle marks the $\sim 0.52^{\circ}$ HAWC error-box for $E>56 \mathrm{TeV}$. Right Panel: Galactic Center spectra fitted with a proton population with a best fit cut-off at $120 \mathrm{PeV}$ (black solid line). The blue, green and red solid lines indicate the 68\% (3.50 PeV), 90\% (2.0 PeV) and 95\% (1.7 PeV) confidence levels for the cut-off, respectively. Both panels adapted from [12].

The Galactic Center is a challenge in a challenge, because it is both a complex region harbouring several potential sources of particle acceleration and can be observed by the ASTRI Mini-Array only at high $\left(\sim 60^{\circ}\right)$ zenith angle for a couple of months in Summer. Currently, the main IACTs detected non-variable emission with no significant cut-off up to a few tens of TeV, making it a very promising PeVatron candidate with hadronic emission. The ASTRI Mini-Array will make use of two major assets. Its large field of view will allow us to map the whole Galactic Center region in a single observation and its excellent angular resolution will help us to identify any high-energy source among several candidates. Figure 2, right panel, shows a $260 \mathrm{hr}$ ASTRI Mini-Array simulation of the Galactic Center, similar in exposure to the H.E.S.S. one [27]. We obtain a 95\% lower limit for a cut-off energy of the hadronic population at $1.7 \mathrm{PeV}$. The details of the simulation and results are given in [12].

Cosmology and fundamental physics. Extra-galactic sources, such as blazars, can successfully probe the level and the evolution of the extra-galactic background light (EBL), one of the most important contribution of absorption of their emission, resulting in an alteration of their spectra 

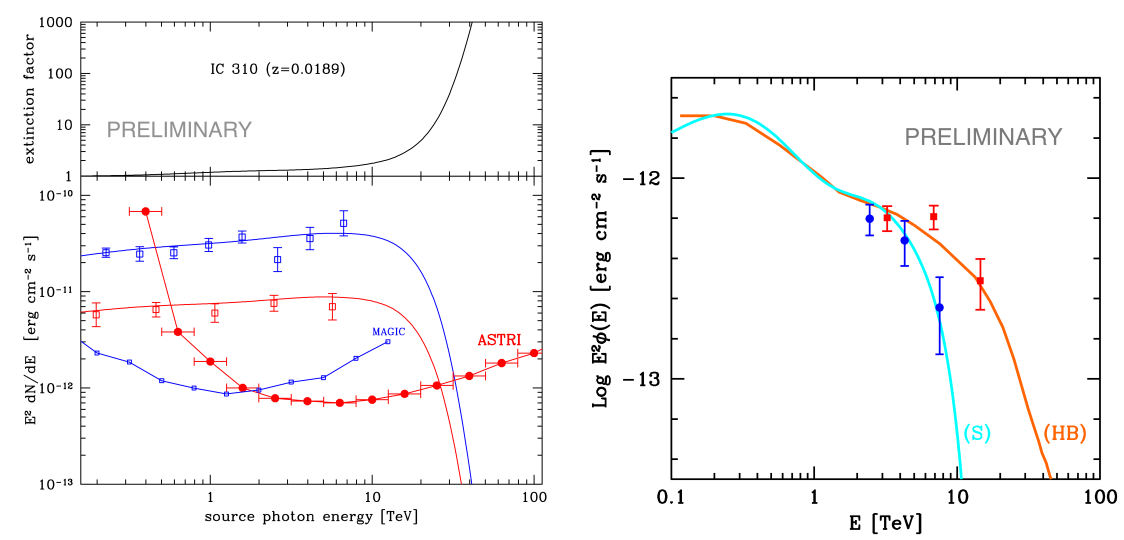

Figure 3: Left Panel: extinction factor for photon-photon interaction on EBL at the IC 310 source distance (upper panel); MAGIC (blue dots) and ASTRI Mini-Array (red dots) 50 hours, $5 \sigma$ on-axis differential sensitivity. Right Panel: Simulated very high-energy spectrum of 1ES 0229+220 for the standard (light blue, $200 \mathrm{hr}$ integration time) and hadron beam (red, $250 \mathrm{hr}$ integration time) scenarios. Both plot adapted from [12].

at energies above a few tens of $\mathrm{GeV}$, with a strong dependence on the source distance. As shown in [28], from the mid-IR to the far-IR, where the IR background intensity is maximal, EBL direct measurements are prevented by the overwhelming dominance of local emission from both the Galaxy and our Solar system. The ASTRI Mini-Array can play a fundamental role in investigating the EBL IR component. From the relation between the energy of the $\gamma$-ray, $E_{\mathrm{TeV}}$, and the wavelength of the target EBL photon, $\lambda_{\max }\left(\lambda_{\max } \simeq 1.24 \times E_{\mathrm{TeV}}[\mu \mathrm{m}]\right)$ we see that we can investigate the (10-30) $\mu \mathrm{m}$ EBL component by investigating blazar spectra in the energy range (10-30) TeV. Best candidates to constrain the EBL up to $\lambda \sim 100 \mu \mathrm{m}$ are low-redshift radio galaxies (such as M 87, IC 310, Centaurus A) and local star-bursting and active galaxies (such as M 82, NGC 253, NGC 1068). Figure 3, left panel, shows in the upper panel the extinction factor at $z=0.0189$ (IC 310 distance), while in the bottom panel the MAGIC (blue dots) and ASTRI Mini-Array (red dots) $50 \mathrm{hr}, 5 \sigma$ on-axis differential sensitivity and two different spectral states for IC 310. We clearly see that even in the lower flux states we can detect this source up to $30 \mathrm{TeV}$.

Blazars' relativistic jets are considered as a possible site of acceleration of ultra high-energy cosmic-rays (UHECRs). The "hadron-beam" scenario can connect UHECRs and blazar jets, by invoking that jets in extreme BL Lac objects could produce hadron beams, i.e., collimated beams of high-energy protons/nuclei. While travelling towards the Earth, UHECRs lose energy through photo-meson and pair production mechanisms, which in turn trigger the development of electromagnetic cascades producing $\gamma$-ray photons and neutrinos. Because of the reduced distance, these $\gamma$-ray photons experience a less severe EBL absorption. The net results is that the observed gamma-ray spectrum extends at energies much higher $(\mathrm{E}>10 \mathrm{TeV})$ than those allowed by the conventional EBL propagation. Figure 3, rigth panel, shows the simulated VHE spectrum of 1ES 0229+220 for the standard leptonic (light blue) and hadron beam (red) scenarios. The ASTRI Mini- Array would be able to obtain a significative detection up to $20 \mathrm{TeV}$, disentangling between the two models by means of a deep $(\approx 250 \mathrm{hr})$ observation. 


\section{Synergies}

The Islas Canarias is a very important astrophysical site. Among the many observing facilities, they host the Telescopio Nazionale Galileo (managed by the Fundación Galileo Galilei-INAF), the Liverpool Robotic Telescope, the Gran Telescopio de Canarias, and several optical telescopes managed by the Instituto de Astrofísica de Canarias. Moreover, the MAGIC Cherenkov array is currently operational at the Roque de los Muchachos Observatory, where the future CTA Northern array will be deployed. Possible synergies with MAGIC and CTAO would allow us to extend the ASTRI Mini-Array spectral performance in the sub-TeV regime, with almost no breaks from a few tens of $\mathrm{GeV}$ up to hundreds of $\mathrm{TeV}$. This opportunity is important also for studies of gamma-ray bursts (GRBs). As demonstrated by MAGIC and H.E.S.S., GRBs may be related to relativistic phenomena observable also at photon energies larger than a few TeV. The LHAASO array detected a dozen of sources with photons up to several hundreds of TeV. Potential synergies are important to make use of the ASTRI Mini-Array angular resolution in combination with the LHAASO extended energy range. In the high-energy band (0.1-100) GeV both AGILE and Fermi satellites will be operational during the ASTRI Mini-Array observing period. At lower energies, there are several SKA precursors both in the Southern hemisphere (MeerKAT and ASKAP) and in the Northern one (LOFAR) which are already obtaining interesting results and will be synergic with the ASTRI Mini-Array. In the immediate future, the Imaging X-ray Polarimetry Explorer (IXPE) will be launched in the Fall 2021, allowing, among many other topics, to shed light on the geometry and the emission mechanism of AGNs and to investigate how particles are accelerated in PWNe.

\section{Conclusions}

The ASTRI Mini-Array will start science operation in 2024, observing the sky above a few TeV and up to $200 \mathrm{TeV}$. For the first time, the ASTRI Mini-Array will join together the very high-energy domain typical of the WCDAs with the precision domain, i.e., the combination of accurate energy and angular resolutions, typical of IACTs. This is unprecedented, and will allow us to shed light on several open questions of very high-energy astrophysics.

\section{Acknowledgments}

This work was conducted in the context of the ASTRI Project. This work is supported by the Italian Ministry of University and Research (MUR) with funds specifically assigned to the Italian National Institute for Astrophysics (INAF). We acknowledge support from the Brazilian Funding Agency FAPESP (Grant 2013/10559-5) and from the South African Department of Science and Technology through Funding Agreement 0227/2014 for the South African Gamma-Ray Astronomy Programme. This work has been supported by H2020-ASTERICS, a project funded by the European Commission Framework Programme Horizon 2020 Research and Innovation action under grant agreement n. 653477. IAC is supported by the Spanish Ministry Ministry of Science and Innovation (MICIU). We also thank the Fundación Galileo Galilei - INAF, Fundación Canaria (FGG) for its support in deploying and managing the ASTRI Mini-Array in Tenerife. 


\section{References}

[1] F. Aharonian, A.G. Akhperjanian, A.R. Bazer-Bachi, M. Beilicke, W. Benbow, D. Berge et al., Observations of the Crab nebula with HESS, A\&A 457 (2006) 899 [astro-ph/0607333].

[2] J. Aleksić, E.A. Alvarez, L.A. Antonelli, P. Antoranz, M. Asensio, M. Backes et al., Performance of the MAGIC stereo system obtained with Crab Nebula data, Astroparticle Physics 35 (2012) 435 [1108 . 1477].

[3] T.C. Weekes, H. Badran, S.D. Biller, I. Bond, S. Bradbury, J. Buckley et al., VERITAS: the Very Energetic Radiation Imaging Telescope Array System, Astroparticle Physics 17 (2002) 221 [astro-ph/0108478].

[4] A.U. Abeysekara, A. Albert, R. Alfaro, C. Alvarez, J.D. Álvarez, R. Arceo et al., Observation of the Crab Nebula with the HAWC Gamma-Ray Observatory, ApJ 843 (2017) 39 [1701. 01778].

[5] Z. Cao, A future project at tibet: the large high altitude air shower observatory (LHAASO), Chinese Physics C 34 (2010) 249.

[6] M. Amenomori, X.J. Bi, D. Chen, T.L. Chen, W.Y. Chen, S.W. Cui et al., Search for Gamma Rays above $100 \mathrm{TeV}$ from the Crab Nebula with the Tibet Air Shower Array and the $100 \mathrm{~m}^{2}$ muon Detector, ApJ 813 (2015) 98.

[7] Cherenkov Telescope Array Consortium, B.S. Acharya, I. Agudo, I. Al Samarai, R. Alfaro, J. Alfaro et al., Science with the Cherenkov Telescope Array (2019), 10.1142/10986.

[8] O. Gueta and the CTA Consortium, The Cherenkov Telescope Array: layout, design and performance, in Proceedings of the $37^{\text {th }}$ ICRC, (2021).

[9] S. Scuderi, The ASTRI Program, in European Physical Journal Web of Conferences, vol. 209 of European Physical Journal Web of Conferences, p. 01001, Sept., 2019, DOI.

[10] E. Giro, R. Canestrari, G. Sironi, E. Antolini, P. Conconi, C.E. Fermino et al., First optical validation of a Schwarzschild Couder telescope: the ASTRI SST-2M Cherenkov telescope, A\&A 608 (2017) A86 [1709.08418].

[11] S. Lombardi, O. Catalano, S. Scuderi, L.A. Antonelli, G. Pareschi, E. Antolini et al., First detection of the Crab Nebula at TeV energies with a Cherenkov telescope in a dual-mirror Schwarzschild-Couder configuration: the ASTRI-Horn telescope, A\&A 634 (2020) A22 [1909.12149].

[12] S. Vercellone and ASTRI Collaboration, ASTRI Mini-Array Core Science at the Observatorio del Teide, JHEAp (in preparation) .

[13] R. Zanin, CTAO Project Scientist, Private Communication, (2021).

[14] L. Antonelli and ASTRI Collaboration, The ASTRI mini-array at Teide Observatory, in Proceedings of the $37^{\text {th }}$ ICRC, (2021). 
[15] S. Lombardi and ASTRI Collaboration, Performance of the ASTRI Mini-Array at the Observatorio del Teide, in Proceedings of the $37^{\text {th }}$ ICRC, (2021).

[16] J. Aleksić, S. Ansoldi, L.A. Antonelli, P. Antoranz, A. Babic, P. Bangale et al., The major upgrade of the MAGIC telescopes, Part II: A performance study using observations of the Crab Nebula, Astroparticle Physics 72 (2016) 76 [1409. 5594].

[17] M. Holler, M. de Naurois, D. Zaborov, A. Balzer and R. Chalmé-Calvet, Photon Reconstruction for H.E.S.S. Using a Semi-Analytical Model, in 34th International Cosmic Ray Conference (ICRC2015), vol. 34 of International Cosmic Ray Conference, p. 980, July, 2015 [1509.02896].

[18] A.U. Abeysekara, R. Alfaro, C. Alvarez, J.D. Álvarez, R. Arceo, J.C. Arteaga-Velázquez et al., The HAWC Real-time Flare Monitor for Rapid Detection of Transient Events, ApJ 843 (2017) 116 [1704.07411].

[19] G. di Sciascio and Lhaaso Collaboration, The LHAASO experiment: From Gamma-Ray Astronomy to Cosmic Rays, Nuclear and Particle Physics Proceedings 279-281 (2016) 166.

[20] A. D'Aì and ASTRI Collaboration, Galactic Science with the ASTRI-Mini Array during the Observatory phase of the project, in Proceedings of the $37^{\text {th }}$ ICRC, (2021).

[21] F. Saturni and ASTRI Collaboration, Extragalactic observatory science with the ASTRI MiniArray at the Observatorio del Teide, in Proceedings of the $37^{\text {th }}$ ICRC, (2021).

[22] MAGIC Collaboration, V.A. Acciari, S. Ansoldi, L.A. Antonelli, A. Arbet Engels, D. Baack et al., Teraelectronvolt emission from the $\gamma$-ray burst GRB 190114C, Nature 575 (2019) 455.

[23] M. Cardillo and ASTRI Collaboration, The ASTRI Mini-Array: a breakthrough in the Cosmic Ray study, in Proceedings of the $37^{\text {th }}$ ICRC, (2021).

[24] Z. Cao, F.A. Aharonian, Q. An, L.X. Axikegu, Bai, Y.X. Bai, Y.W. Bao et al., Ultrahighenergy photons up to 1.4 petaelectronvolts from $12 \gamma$-ray Galactic sources, Nature 594 (2021) 33.

[25] E. Aliu, S. Archambault, T. Aune, B. Behera, M. Beilicke, W. Benbow et al., Investigating the TeV Morphology of MGRO J1908+06 with VERITAS, ApJ 787 (2014) 166 [1404 . 7185].

[26] A.U. Abeysekara, A. Albert, R. Alfaro, J.R. Angeles Camacho, J.C. Arteaga-Velázquez, K.P. Arunbabu et al., Multiple Galactic Sources with Emission Above $56 \mathrm{TeV}$ Detected by HAWC, PRL 124 (2020) 021102.

[27] H. E. S. S. Collaboration, H. Abdalla, A. Abramowski, F. Aharonian, F. Ait Benkhali, A.G. Akhperjanian et al., Characterising the VHE diffuse emission in the central 200 parsecs of our Galaxy with H.E.S.S., A\&A 612 (2018) A9 [1706. 04535].

[28] A. Franceschini, G. Rodighiero and M. Vaccari, Extragalactic optical-infrared background radiation, its time evolution and the cosmic photon-photon opacity, A\&A 487 (2008) 837 [0805. 1841]. 